\title{
Simulation of transverse modes with their intrinsic Landau damping for bunched beams in the presence of space charge
}

\author{
Alexandru Macridin, Alexey Burov, Eric Stern, James Amundson, and Panagiotis Spentzouris \\ Fermilab, P.O. Box 500, Batavia, Illinois 60510, USA
}

(Received 29 May 2015; published 22 July 2015)

\begin{abstract}
Transverse dipole modes in bunches with space charge are simulated using the SYNERGIA accelerator modeling package and analyzed with dynamic mode decomposition. The properties of the first three space charge modes, including their shape, damping rates, and tune shifts are described over the entire range of space charge strength. The intrinsic Landau damping predicted and estimated in 2009 by one of the authors is confirmed with a reasonable scaling factor of $\simeq 2.4$. For the $\mathrm{KV}$ distribution, very good agreement with PATRIC simulations performed by Kornilov and Boine-Frankenheim is obtained.
\end{abstract}

DOI: 10.1103/PhysRevSTAB.18.074401

PACS numbers: 29.20.D-

\section{INTRODUCTION}

The Landau damping effect, which provides an important mechanism for stabilizing beam propagation, is an important research topic in accelerator physics. The mechanism operates via an energy exchange between the coherent mode and particles resonating with the mode. Thus, for Landau damping to take place, a continuous incoherent spectrum around the coherent frequency is required. Aside from nonlinear lattice elements, incoherent tune spread is also provided by space charge. The damping mechanism caused by space charge is not fully known, especially for bunched beams and at intermediate space charge strength. Many high-intensity hadronic synchrotrons operate in the intermediate or strong space charge regimes (e.g., the Fermilab Booster and the CERN PS). In this paper we neglect nonlinear lattice effects and focus only on the intrinsic effect of space charge on the transverse dipole modes. The beam dynamics of a bunch is investigated over the entire range of the strength of the space charge effect, from no space charge to the strong space charge limit. A comparison between the modes' properties between bunches with a transverse Gaussian distribution (3D-G) and bunches with a transverse Kapchinsky-Vladimirsky (KV-G) distribution, both with a longitudinally Gaussian profile, is also presented.

It was shown long ago that without lattice nonlinearities space charge does not contribute to Landau damping in coasting beams regardless of the density of the resonant particles and the coherent tune shift $[1,2]$. In other words, there is no intrinsic Landau damping for coasting beams. Balbekov [3] addressed the space charge effect on Landau damping in the weak intensity regime and found a damping

Published by the American Physical Society under the terms of the Creative Commons Attribution 3.0 License. Further distribution of this work must maintain attribution to the author(s) and the published article's title, journal citation, and DOI. rate proportional to the incoherent tune shift and independent of the synchrotron tune, which is similar to our results. However his suggestion of a damping rate decreasing with increasing mode number seems to contradict the typical behavior of Landau damping in physical systems [4-6]. The effect of space charge on the modes in bunched beams was also addressed in 1998 by Blaskiewicz [7], who showed that space charge can suppress the fast head-tail instability. He also suggested a solvable square-well air-bag model to address the problem. Head-tail modes in the strong space charge regime were addressed analytically by one of the authors in 2009 [6]. In that work, a general equation describing head-tail modes valid for arbitrary rf buckets, beam distribution functions, and dipole/ quadrupole wake fields was derived. It was also shown, contrary to the coasting beam case, that intrinsic Landau damping is different from zero for bunched beams; a formula estimating the head-tail modes damping rates, with an accuracy limited to a numerical factor with a magnitude of order of unity, was provided for the strong space charge regime. Using a different analytical approach, head-tail modes with space charge were also addressed in Ref. [8] for zero wakes and a parabolic potential well, but no quantitative estimation of the Landau damping was derived.

The estimate of the intrinsic Landau damping rates at strong space charge [6] and of zero damping at zero space charge suggest that the maximal damping rate occurs in the intermediate space charge region. However, the detailed behavior of the damping rates for the entire range of the space charge strength is poorly known. Employing the PATRIC code, Kornilov and Boine Frankenheim [9] ran particle tracking simulations which address the collective modes for bunches with transverse $\mathrm{KV}$ and longitudinally Gaussian distributions (KV-G). They provide the intrinsic damping rates for the first two modes for a wide range of space charge strengths. The modes' damping rates are extracted from the long-time behavior of the momentum 
functions defined as projections of the simulated dipole moment distribution on the theoretical strong space charge mode shapes. The implicit assumption behind this approach is that after a long enough time after the beam is excited with an approximate mode shape only one mode survives, the others being exponentially damped.

In this paper we study the collective modes for $3 \mathrm{D}-\mathrm{G}$ and $\mathrm{KV}-\mathrm{G}$ bunches. In the case of intrinsic Landau damping for the KV-G bunches, we demonstrate an impressively good agreement with Ref. [9]. For the 3D-G bunch, the very recent report of our colleagues in Ref. [10] shows an order of magnitude difference between our intrinsic damping values and theirs. While our simulations in the strong space charge regime yield a damping behavior as a function of the space charge strength in agreement with the theoretical predictions [6], their estimation does not. However, we hope that the agreement between our simulations will be significantly improved when more macroparticles and wider Gaussian tails are considered in their simulations [11].

For this study we employ the SYNERGIA $[12,13]$ accelerator modeling package to simulate the propagation of a single Gaussian beam through a linear lattice. Space charge is calculated with a 3D Poisson solver. The phase space density of $10^{7}$ macroparticles propagating through a linear lattice consisting of drift, quadrupole, and rf cavity elements is stored every turn. The evolution of the phase space density is analyzed using the dynamic mode decomposition technique [14-17].

Dynamic mode decomposition (DMD) is a data-driven algorithm used for modal analysis and model reduction in both linear and nonlinear systems. Developed initially by the fluid mechanics community to address turbulent flows, it has proven to be a successful technique in many different areas [18-20]. One important feature of this method is the direct calculation of mode dynamics. Dynamic properties of data are poorly addressed by principal component analysis (PCA), a widely used modal decomposition method. PCA determines the spatially coherent structures from the time-averaged correlation matrix, thus implicitly assuming stationary data. In contrast, DMD considers both spatial and temporal orthogonalities, calculating mode shape, frequency, and growth/damping rates. To our knowledge this is the first investigation of beam dynamics with DMD.

Whereas the long-time behavior of the momentum functions allows only limited access to the mode properties, the DMD method allows a full extraction of the mode properties, even when multiple modes with similar damping rates are excited simultaneously. We are able to visualize transverse mode shapes in phase space as they evolve from the rotationally symmetric shapes characteristic of the noninteracting problem to the momentumindependent shapes characteristic of the strong space charge limit. At large space charge the mode shapes and tunes are in good agreement with theoretical predictions [6]. The damping rate dependence on space charge strength and mode number is also in agreement with the analytical estimates. The large difference in the damping magnitude between the 3D-G and the KV-G bunches shows that Landau damping is very sensitive to the bunch transverse distribution. This is not surprising, since the resonant particles are the ones residing in both the longitudinal and the transverse tails. We propose formulas for the damping rates and tune shifts which fit the numerical data reasonably well over the entire range of the space charge strength.

The paper is organized as follows. The problem is defined in Sec. II. The DMD method is introduced in Sec. III. The Synergia simulation is discussed in Sec. IV. The results of the mode analysis for 3D-G bunches are presented in Sec. VA. A comparison of modes' properties between 3D-G and KV-G bunches is given in Sec. V B. Simulation challenges are discussed in Sec. VI, and conclusions are drawn in Sec. VII.

\section{FORMALISM}

In this paper we investigate the relevant modes that characterize the dynamics of the transverse displacement density, defined as

$X(z, u, s)=\frac{\int d x d p_{x} d y d p_{y} x \rho\left(x, p_{x}, y, p_{y}, z, u, s\right)}{\rho(z, u, s)}$,

where $s$ is the distance along the reference trajectory, $z$ is the longitudinal position relative to the reference particle, and $u=\frac{\delta p}{p}$ is the relative momentum spread. $\rho\left(x, p_{x}, y, p_{y}, z, u, s\right)$ is the density in $6 D$ phase space and

$$
\rho(z, u, s)=\int d x d p_{x} d y d p_{y} \rho\left(x, p_{x}, y, p_{y}, z, u, s\right)
$$

is the density in the longitudinal plane.

The relevant parameter governing the transverse mode dynamics is the space charge parameter, defined as the ratio of the incoherent tune shift, $\Delta Q_{s c}$, and the synchrotron tune, $Q_{s}$. The equation of motion for the transverse displacement of a particle experiencing a transverse space charge force $\frac{e E_{x}}{\gamma^{2}}$ is

$$
\frac{d^{2} x_{i}}{d s^{2}}+\frac{\omega^{2} Q_{\beta}^{2}}{\beta^{2} c^{2}} x_{i}=\frac{e E_{x}}{m \gamma^{3} \beta^{2} c^{2}},
$$

where $\omega$ is the angular revolution frequency and $Q_{\beta}$ is the betatron tune; the smooth approximation was assumed. With a substitution $\beta c / \omega Q_{\beta} \rightarrow \beta(s)$, the incoherent space charge tune shift for infinitely-small amplitude particles follows: 


$$
\Delta Q_{\mathrm{sc}}=\frac{e}{m \gamma^{3} \beta^{2} c^{2}} \frac{1}{4 \pi} \oint \beta(s) \frac{\partial E_{x}}{\partial x}(\bar{x}, s) d s,
$$

where $\beta(s)$ is the lattice beta function and $\bar{x}$ is the beam center coordinate. In general $\Delta Q_{\mathrm{sc}}$ depends on a particle's transverse amplitude and longitudinal coordinate. Therefore for the definition of the space charge parameter it is more convenient to use the effective incoherent tune shift, $\Delta Q_{\text {sc eff }}$, determined by integrating over the transverse degrees of freedom when calculating the equation for the eigenfunctions [6]. $\Delta Q_{\text {sc eff }}$ depends on the transverse beam profile; the space charge parameter can be written as

$$
q_{\mathrm{eff}}=0.52 \frac{\Delta Q_{\mathrm{sc} \max }}{Q_{s}}
$$

for the 3D-G beam and

$$
q_{\mathrm{eff}}=\frac{\Delta Q_{\mathrm{sc} \max }}{Q_{s}}
$$

for the KV-G beam. $\Delta Q_{\text {sc max }}$ is the maximum tune shift measured at the bunch center; it is calculated numerically by employing Eq. (4) and using the electric field as determined by the simulation.

In a purely linear lattice, the zero mode, defined by a uniform transverse displacement of the bunch, i.e., $X(z, u, s)=X(s)$, is undamped regardless of the value of the space charge parameter. This is a consequence of the fact that the space charge forces are internal bunch forces. To prove that, we write the equation of motion for the particle $i$ transverse displacement as

$$
\frac{d^{2} x_{i}}{d s^{2}}+\frac{\omega^{2} Q_{\beta}^{2}}{\beta^{2} c^{2}} x_{i}=\sum_{j} F\left(x_{j}-x_{i}, y_{j}-y_{i}, z_{j}-z_{i}\right),
$$

where $F\left(x_{j}-x_{i}, y_{j}-y_{i}, z_{j}-z_{i}\right)$ is proportional to the Coulomb force of particle $j$ acting on particle $i$. A uniform displacement of all the particles

$$
\tilde{x}_{i}(s)=x_{i}(s)+X(s),
$$

corresponds to the mode with $X(z, u, s)=X(s)$ [see Eq. (1)]. The equation of motion for $\tilde{x}_{i}(s)$ can be written as

$$
\begin{aligned}
& \frac{d^{2} \tilde{x}_{i}}{d s^{2}}+\frac{\omega^{2} Q_{\beta}^{2}}{\beta^{2} c^{2}} \tilde{x}_{i}=\frac{d^{2} x_{i}}{d s^{2}}+\frac{\omega^{2} Q_{\beta}^{2}}{\beta^{2} c^{2}} x_{i}+\frac{d^{2} X}{d s^{2}}+\frac{\omega^{2} Q_{\beta}^{2}}{\beta^{2} c^{2}} X \\
& \quad=\sum_{j} F\left(\tilde{x}_{j}-\tilde{x}_{i}, y_{j}-y_{i}, z_{j}-z_{i}\right) .
\end{aligned}
$$

Since the space charge force depends only on the relative distance between particles, i.e., $F\left(\tilde{x}_{j}-\tilde{x}_{i}, y_{j}-y_{i}, z_{j}-z_{i}\right)=$ $F\left(x_{j}-x_{i}, y_{j}-y_{i}, z_{j}-z_{i}\right)$, it follows that

$$
\frac{d^{2} X}{d s^{2}}+\frac{\omega^{2} Q_{\beta}^{2}}{\beta^{2} c^{2}} X=0
$$

showing that the zero mode is undamped and oscillates with the tune $Q=Q_{\beta}$ regardless of the value of the space charge parameter.

In case of zero space charge the solution for the transverse displacement, $X(z, u)=X(z, u, s) e^{i \frac{\omega Q}{\beta c} s}$, reads [21]

$$
X(z, u) \equiv X(r, \theta)=R(r) e^{i m \theta},
$$

with $r$ and $\theta$ being the amplitude and the phase of the synchrotron oscillation, respectively. The tune shift is given by

$$
Q-Q_{\beta}=m Q_{s}
$$

for integer $m$. The modes are defined by the angular number $m$ and are radially degenerate.

According to Ref $[6,8]$, large $q_{\text {eff }}$ leads to solutions which are weakly dependent on $u$, i.e., $X(z, u) \approx Y(z)$. Throughout this paper we will refer to the space charge modes in the large $q_{\text {eff }}$ limit as space charge harmonics. The space charge harmonics, calculated in Ref [6], form an orthogonal set

$$
\int Y_{k}(z) Y_{m}(z) \rho(z)=\delta_{k m},
$$

where $k$ and $m$ represent mode numbers. For the mode $k$ the tune shift is $\frac{\nu_{k} Q_{s}}{q_{\text {eff }}}\left(\nu_{k}\right.$ are numerical coefficients calculated in Ref [6]), while the Landau damping is $\lambda_{k} T_{0} \simeq \frac{k^{4} 2 \pi Q_{s}}{q_{\text {eff }}^{3}} \cdot \lambda_{k}$ represents the mode $k$ damping rate and $T_{0}$ is the revolution period [22]. The results of our simulations at large $q_{\text {eff }}$ are shown to be in agreement with these theoretical results.

\section{DYNAMIC MODE DECOMPOSITION}

Dynamic mode decomposition [14-17] is a new technique used successfully for mode analysis and model reduction in many fields such as fluid mechanics [18], neuroscience [19], video streaming, and pattern recognition [20]. There is a significant number of papers dedicated to the method. Here we present only a short description of it.

Suppose that as a result of a simulation or an experiment the following set of data is obtained:

$$
X\left(q, t_{0}\right), X\left(q, t_{1}\right), \ldots, X\left(q, t_{N+1}\right),
$$

where $X\left(q, t_{i}\right)$ is an $M$ dimensional vector and $t_{i}$, $i=\overline{1, N+1}$ represent equidistant time slices, i.e., $t_{i}=i \Delta t$. DMD assumes that the data evolution in time can be approximated by a linear operator $A$ $(M \times M)$ such as 


$$
A X\left(q, t_{i}\right)=X\left(q, t_{i+1}\right),
$$

for all $i=\overline{1, N}$. If the eigenvectors and the eigenvalues of $A$ are given by

$$
A v_{j}=\mu_{j} v_{j},
$$

then one can write

$$
X\left(q, t_{i}\right)=A^{i} X\left(q, t_{0}\right)=\sum_{j} \mu_{j}^{i} \alpha_{j} v_{j}=\sum_{j} \mu_{j}^{i} \phi_{j}
$$

By writing

$$
\mu_{j}=e^{-\lambda_{j} \Delta t} e^{i \omega_{j} \Delta t},
$$

it becomes evident that this expansion provides the damping rates $\lambda_{j}$ and the frequencies $\omega_{j}$ for each mode $\phi_{j}$.

Seeking a linear operator to approximate the data does not imply that the method can be applied only to linear dynamics. The linear operator $A$ can be considered a regression solution to the nonlinear dynamics. A more rigorous argument for applying DMD to nonlinear dynamics is made by showing that DMD can be considered a numerical approximation of Koopman spectral analysis [15-17].

DMD is a technique that computes the eigenvalues and the eigenvectors of $A$ for the given set of data. Defining the $M \times N$ matrices $X_{0}$ and $X_{1}$

$$
\begin{aligned}
& X_{0}=\left[\begin{array}{llll}
X\left(q, t_{0}\right) & X\left(q, t_{1}\right) & \ldots & X\left(q, t_{N}\right)
\end{array}\right], \\
& X_{1}=\left[\begin{array}{llll}
X\left(q, t_{1}\right) & X\left(q, t_{2}\right) & \ldots & X\left(q, t_{N+1}\right)
\end{array}\right],
\end{aligned}
$$

the operator $A$ is required to satisfy

$$
A X_{0}=X_{1}
$$

In the DMD approximation $A$ is

$$
A=X_{1} X_{0}^{+},
$$

where $X_{0}^{+}$is the pseudoinverse of $X_{0}$. The $A$ given by Eq. (22) is not always a solution to Eq. (21), since the columns of $X_{0}$ are not guaranteed to be linearly independent. However it can be shown that this choice of $A$ minimizes $\left\|A X_{0}-X_{1}\right\|[16]$.

The DMD solution for finding $A$ is to project the problem onto the space spanned by the singular value decomposition (SVD) modes of $X_{0}$. The DMD algorithm includes the following steps:
1. Compute the SVD of $X_{0}$,

$$
X_{0}=U \Sigma V^{*}
$$

2. Project $A$ onto the space spanned by the SVD states

$$
\tilde{A}=U^{*} A U=U^{*} X_{1} V \Sigma^{-1} .
$$

Note that $\tilde{A}$ is reduced to rank $r$, where $r$ is equal to the number of the SVD states of $X_{0}$.

3. Solve the eigenvalue problem in the projected space

$$
\tilde{A} w_{j}=\mu_{j} w_{j}
$$

4. The DMD eigenmodes are

$$
\Phi_{j}=U w_{j}
$$

It is easy to check that $A \phi_{j}=A U w_{j}=U \tilde{A} w_{j}=\mu_{j} U w_{j}=$ $\mu_{j} \phi_{j}$.

The number of DMD modes is given by the rank of $X_{0}$; the DMD modes are a linear combination of the SVD modes.

DMD was developed for fluid dynamics analysis where typically $M \gg N$. For bunch mode analysis often $M<N$, which implies that the columns of $X_{0}$ are not linearly independent. In this case a direct application of the DMD algorithm presented above can produce misleading results. The solution is to augment each data slice with subsequent time step data slices [19]

$Z_{0}=\left[\begin{array}{cccc}X\left(q, t_{0}\right) & X\left(q, t_{1}\right) & \ldots & X\left(q, t_{N-p}\right) \\ X\left(q, t_{1}\right) & X\left(q, t_{2}\right) & \ldots & X\left(q, t_{N-p+1}\right) \\ \cdot & & & \\ \cdot & & & \\ X\left(q, t_{p}\right) & X\left(q, t_{p+1}\right) & \ldots & X\left(q, t_{N}\right)\end{array}\right]$,

$Z_{1}=\left[\begin{array}{cccc}X\left(q, t_{1}\right) & X\left(q, t_{2}\right) & \ldots & X\left(q, t_{N-p+1}\right) \\ X\left(q, t_{2}\right) & X\left(q, t_{3}\right) & \ldots & X\left(q, t_{N-p+2}\right) \\ \cdot & & & \\ \cdot & & & \\ X\left(q, t_{p+1}\right) & X\left(q, t_{p+2}\right) & \ldots & X\left(q, t_{N+1}\right)\end{array}\right]$,

and apply the DMD method for the problem $A Z_{0}=Z_{1}$, where now $A$ is a $(p+1) M \times(p+1) M$ operator describing the evolution of the augmented data. 


\section{SIMULATIONS}

A simple lattice made by 10 identical OFORODO (driftfocusing quad-drift-rf cavity-drift-defocusing quad-drift) cells was chosen for our investigation. The dynamics is studied using the SYNERGIA accelerator simulation package $[12,13]$. The second-order split-operator method of Yoshida [23] is used to address space charge effects. Beam propagation consists of interleaved sequences of single-particle propagation followed by a space charge kick, followed by single-particle propagation followed by space charge kick, etc. In order to focus only on the intrinsic Landau damping, the single particle propagation is realized using first order maps. Thus the single particle dynamics is purely linear. The vertical and horizontal betatron tunes are set equal as are the vertical and horizontal beam emittances. The chromaticity is zero and the longitudinal potential is parabolic.

The space charge kicks are proportional to the electric field in the beam frame. The field itself is calculated by numerically solving the Poisson equation. For that purpose we use a 3D solver with open boundary conditions [24]. We take extreme care to ensure that the numerical approximations involved do not introduce spurious forces between particles. Charge deposition on the grid and field interpolation from the grid to the particle's position is done in such a way that the sum of the reciprocal forces between any two particles is zero. When this condition is not rigorously enforced the modes are overdamped. This happens especially at large $q_{\text {eff }}$ where potential spurious numerical effects would be larger.

To study the modes, the bunch distribution in phase space is initially excited with different shapes. Each macroparticle's transverse coordinate $x_{i}$ is changed by

$$
\Delta x_{i}=-a f\left(z_{i}, u_{i}\right)
$$

The change in the phase-space density is

$$
\Delta \rho\left(x, p_{x}, y, p_{y}, z, u\right)=-a f(z, u) \frac{\partial \rho}{\partial x},
$$

while the initial transverse dipole is proportional to the applied excitation shape $f(z, u)$,

$$
X(z, u)=-a f(z, u) \int d x x \frac{\partial \rho}{\partial x}=a f(z, u)
$$

$a$ is the excitation amplitude; it must be taken small enough to ensure the beam dynamics is in the linear damping regime. In our simulations $a \approx 0.03 \sigma_{x}$ at small $q_{\text {eff }}$ and $a \approx 0.005 \sigma_{x}$ at large $q_{\text {eff }}$.

The DMD technique described in Sec. III is applied to the data set $X\left(z, u, s_{n}\right)$ defined by Eq. (1) and produced by the SYNERGIA simulation.

\section{RESULTS}

\section{A. Transverse Gaussian distribution (3D-G)}

The zero mode corresponds to the transverse displacement of the beam as a whole, i.e., $X(z, u)$ is constant. Without wake fields and lattice nonlinearities, its dynamics is reduced to undamped betatron oscillation [see Eq. (10)].

To validate our simulations we start in the strong space charge regime, where a comparison with theoretical results can be done. In Figs. 1(a)-(d) we show the spatial distribution of the first four modes at large $q_{\text {eff }}$. For each mode the beam is excited with a shape proportional to the corresponding space charge harmonic calculated in Ref. [6]. In agreement with the theory we find that the modes' spatial distribution is nearly independent of the momentum coordinate $u$. In Figs. 1(e)-(h) we compare the modes' dependence on $z$ with the theoretical prediction. The agreement is very good.
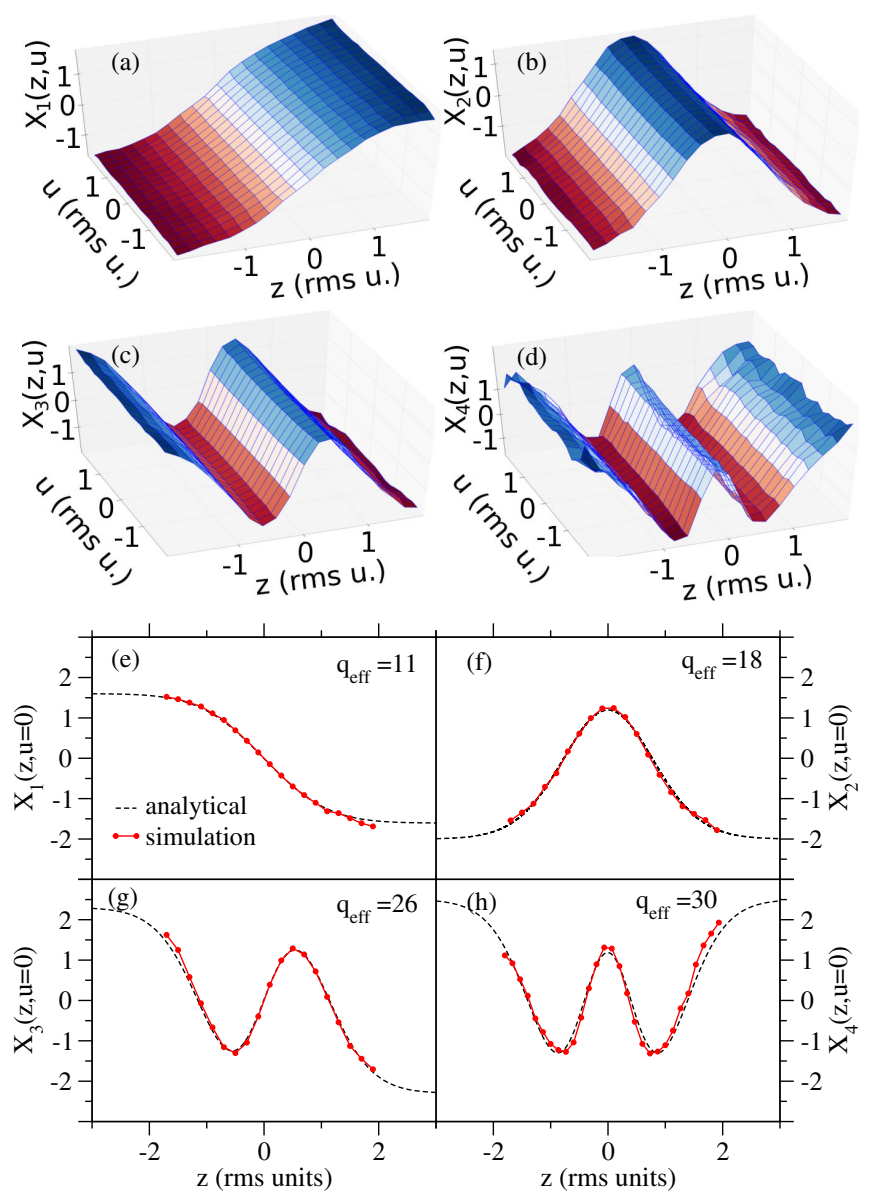

FIG. 1. The first four modes of a 3D-G bunch in the strong space charge regime. (a)-(d) At large $q_{\text {eff }}$ the modes' spatial distribution is nearly independent of $u$, i.e., $X(z, u) \approx Y(z)$, as predicted by Ref. [6]. (e)-(h) Comparison of the simulated modes (solid red) with the theoretical space charge harmonics (dashed black) [6]. The agreement is very good. 


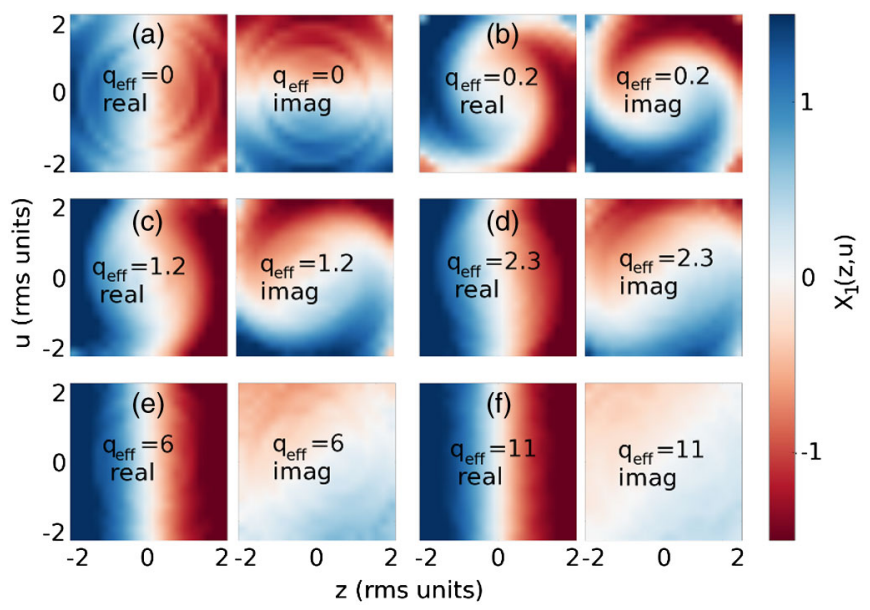

FIG. 2. 3D-G bunch. (a)-(f) The mode 1 longitudinal distribution, $X_{1}(z, u)$, for different values of the space charge parameter $q_{\text {eff. }}$. Without space charge, $X_{1}(z, u) \propto e^{i \theta}$. With increasing $q_{\text {eff }}, X_{1}(z, u)$ transforms gradually to the first space charge harmonic [see Fig. 1(a)]. At large $q_{\text {eff }} X_{1}(z, u)$ can be described by a purely real function.

As discussed in Sec. II, for zero space charge the modes are degenerate with respect to radial distribution. The space charge forces lift this degeneracy and the weakly interacting regime is characterized by a large number of modes with tune shifts close to integer multiples of $Q_{s}$. Any excitation, unless proportional to an exact mode shape, will excite a large number of those modes. In order to study the evolution of the strong space charge modes into the weakly interacting regime we excite the beam with excitations proportional to space charge harmonics. Among the many excited modes, we choose for investigation the ones which have the largest overlap with the space charge harmonics.

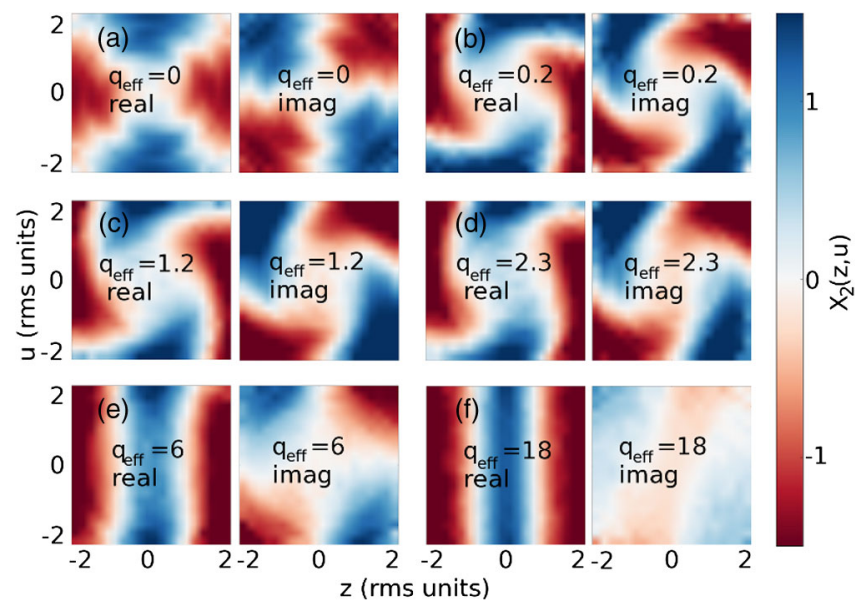

FIG. 3. 3D-G bunch. (a)-(f) The mode 2 longitudinal distribution, $X_{2}(z, u)$, for different values of the space charge parameter $q_{\text {eff }}$. Without space charge, $X_{2}(z, u) \propto e^{i 2 \theta}$. With increasing $q_{\text {eff }}, X_{2}(z, u)$ transforms gradually to the second space charge harmonic [see Fig. 1(b)]. At large $q_{\mathrm{eff}} X_{2}(z, u)$ can be described by a purely real function.

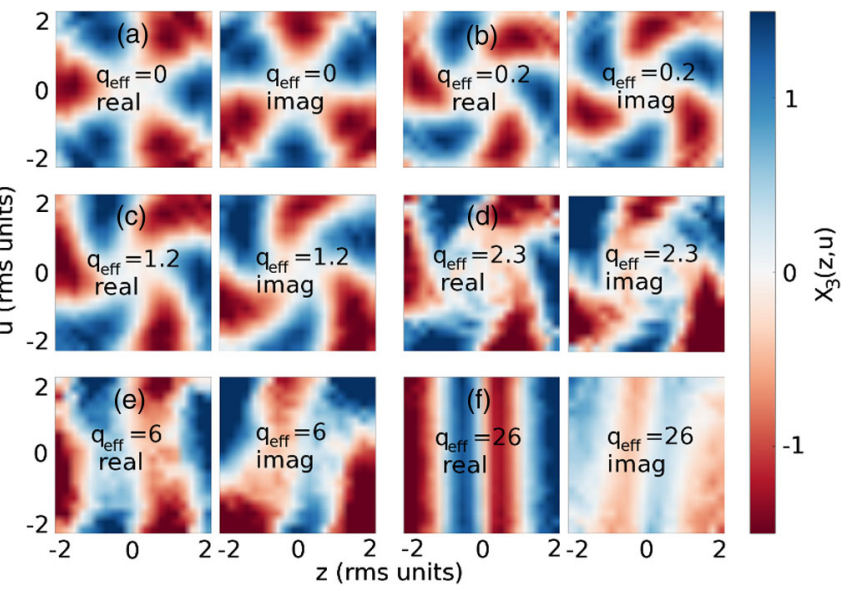

FIG. 4. 3D-G bunch. (a)-(f) The mode 3 longitudinal distribution, $X_{3}(z, u)$, for different values of the space charge parameter $q_{\text {eff }}$. Without space charge, $X_{3}(z, u) \propto e^{i 3 \theta}$. With increasing $q_{\text {eff }}, X_{3}(z, u)$ transforms gradually to the third space charge harmonic [see Fig. 1 (c)]. At large $q_{\text {eff }} X_{3}(z, u)$ can be described by a purely real function.

These are also the modes that change smoothly when going from large to small $q_{\text {eff }}$.

In Figs. 2, 3 and 4 we show the spatial distribution in longitudinal phase space for the modes 1, 2, and 3, respectively, for different values of $q_{\text {eff }}$. When $q_{\text {eff }}=0$ the modes are characterized by the angular number $m=k$ (where $k$ is the mode number), i.e., $X_{k}(z, u) \equiv X_{k}(r, \theta)=$ $R_{k}(r) e^{i k \theta}$. With increasing $q_{\text {eff }}$ the modes transform gradually into the space charge harmonics. While at small $q_{\text {eff }}$ the real and the imaginary part of the modes have comparable magnitudes, at large $q_{\text {eff }}$ the modes can be described by purely real functions.

The spatial overlap of the mode $X_{k}(z, u)$ with the space charge harmonic $Y_{k}(z)$

$$
\left\langle X_{k} Y_{k}\right\rangle=\int X_{k}^{*}(z, u) Y_{k}(z) \rho(z, u) d z d u
$$

as a function of $q_{\text {eff }}$ is presented in Fig. 5. At small $q_{\text {eff }}$ the overlap increases rapidly with increasing $q_{\text {eff }}$ and saturates at $q_{\text {eff }} \approx 4 k$ indicating the transition to the strong space charge regime.

The Landau damping for the first three modes is shown in Fig. 6. All three modes display a similar behavior. At small $q_{\text {eff }}$ the damping increases quickly with increasing $q_{\text {eff }}$. The damping reaches its maximum in the intermediate region around $q_{\mathrm{eff}} \approx 2 k$. The maximum damping increases with the mode number. For $q_{\text {eff }}>4 k$ the damping $\lambda_{k} T_{0} \approx 2.4 \frac{k^{4} 2 \pi Q_{s}}{q_{\text {eff }}^{3}}$. This dependence is in good agreement with the theoretical predictions of Ref. [6]. Note that in Ref. [6] the Landau damping was derived with an accuracy limited by a proportionality factor with the magnitude of order unity. As simulations with KV-G beams show (see Sec. V B), the proportionality factor is sensitive to the 


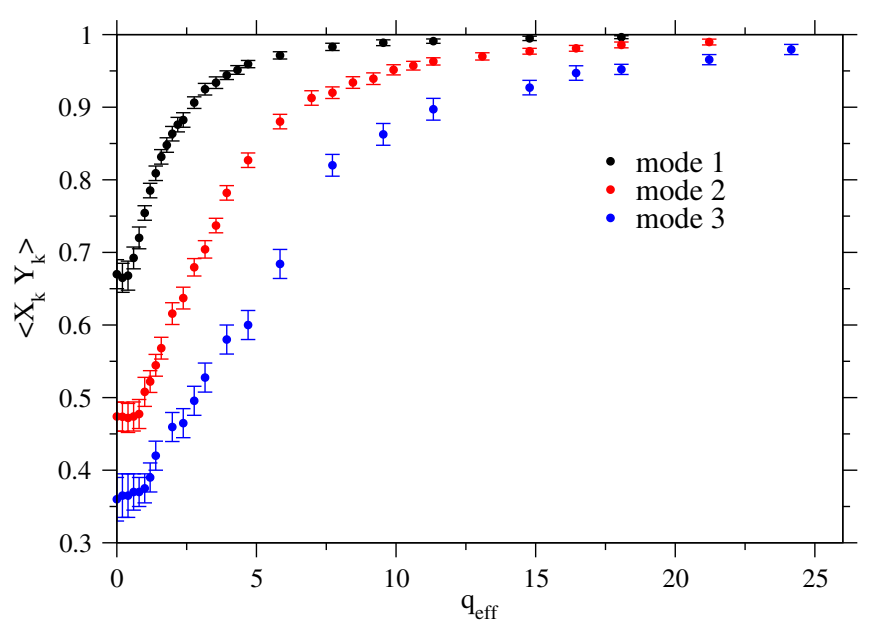

FIG. 5. 3D-G bunch. The overlap $\left\langle X_{k} Y_{k}\right\rangle$ [see Eq. (32)] in the longitudinal phase space of the mode $k$ with the space charge harmonic $k$ for $k=1,2,3$. At small $q_{\text {eff }}$ the overlap increases rapidly with increasing $q_{\mathrm{eff}}$. For $q_{\mathrm{eff}}>\approx 4 k$ the increase of $\left\langle X_{k} Y_{k}\right\rangle$ with $q_{\text {eff }}$ saturates indicating the transition to the strong space charge regime.

transverse bunch distribution. The value of 2.4 is characteristic of transverse Gaussian beams.

We propose a formula for the intrinsic Landau damping of the 3D-G bunches that fits reasonably well over the entire range of space charge strengths (green lines in Fig. 6),

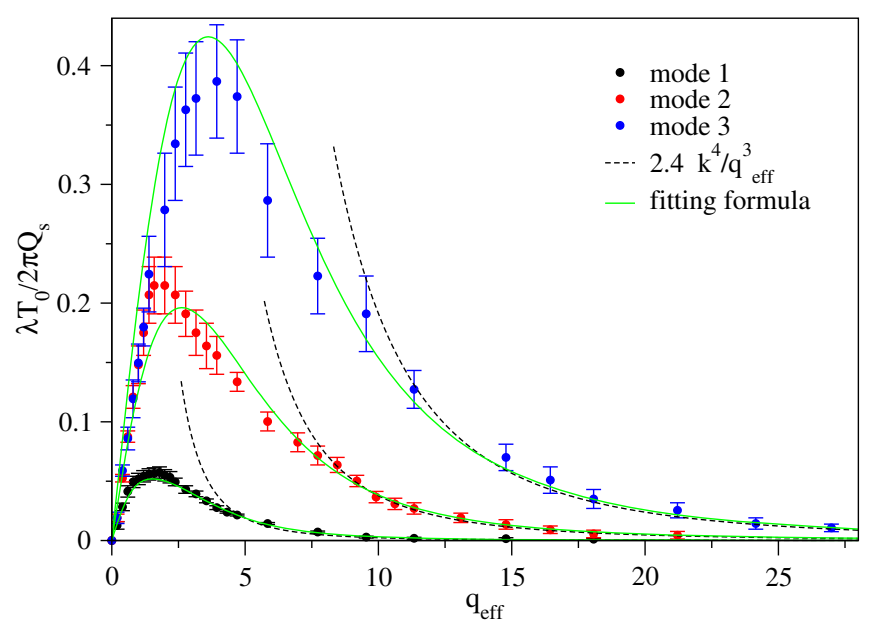

FIG. 6. 3D-G bunch. The Landau damping for modes 1,2 , and 3 versus the space charge parameter $q_{\text {eff }}$. At small $q_{\text {eff }}$ the damping increases quickly with increasing $q_{\text {eff }}$. In the strong space charge regime, $q>\approx 4 k$, we find that $\lambda T_{0} \approx 2.4 \frac{k^{4} 2 \pi Q_{s}}{q_{\text {eff }}^{3}}$, where $k$ is the mode number (dashed lines). This behavior is in agreement with the theoretical predictions [6]. The proportionality factor of 2.4 is characteristic of transverse Gaussian beams. The damping rates of all three modes can be fitted reasonably well for the entire range of the space charge strength by employing Eq. (33) (green lines).

$$
\frac{\lambda_{k} T_{0}}{2 \pi Q_{s}}=\frac{A k q_{\mathrm{eff}}}{1+B\left(\frac{q_{\text {eff }}}{2 k}\right)^{2}+C\left(\frac{\left(q_{\text {eff }}\right.}{2 k}\right)^{3}+k D\left(\frac{q_{\text {eff }}}{2 k}\right)^{4}},
$$

with $A=0.072, B=2.4, C=-1$, and $D=0.48$. It is interesting to note that the data for the first three modes can be fitted with only four coefficients. Inductive reasoning suggests that Eq. (33) can also be used to describe higher order modes; however, the validity of Eq. (33) for $k \geq 4$ remains to be checked by future investigations.

In Fig. 7 the tune shifts for the first three modes as a function of $q_{\text {eff }}$ are shown. Starting from the noninteracting value of $Q_{k}=Q_{\beta}+k Q_{s}$, the tune is suppressed with increasing $q_{\text {eff }}$. In the strong space charge regime $\frac{Q_{k}-Q_{\beta}}{Q_{s}} \approx$ $\frac{\nu_{k}}{q_{\text {eff }}}$ in good agreement with the theoretical estimate of Ref. [6]. The $\nu_{k}$ are coefficients specific to each mode and were calculated in Ref. [6]. For the first three modes we have $\nu_{1}=1.4, \nu_{2}=4.40$, and $\nu_{3}=8.9$. We propose a fitting formula for the tune shift for the entire range of the space charge strength (green lines in Fig. 7),

$$
\frac{Q_{k}-Q_{\beta}}{Q_{s}}=A_{k} \frac{q_{\mathrm{eff}}}{2}+\sqrt{\left(A_{k} \frac{q_{\mathrm{eff}}}{2}\right)^{2}+k^{2}},
$$

with

$$
A_{k}=\frac{k^{2}}{\nu_{k}}
$$

For the first three modes $A_{1}=0.72, A_{2}=0.91$, and $A_{3}=1.01$. Equation (34) is obtained by modifying the

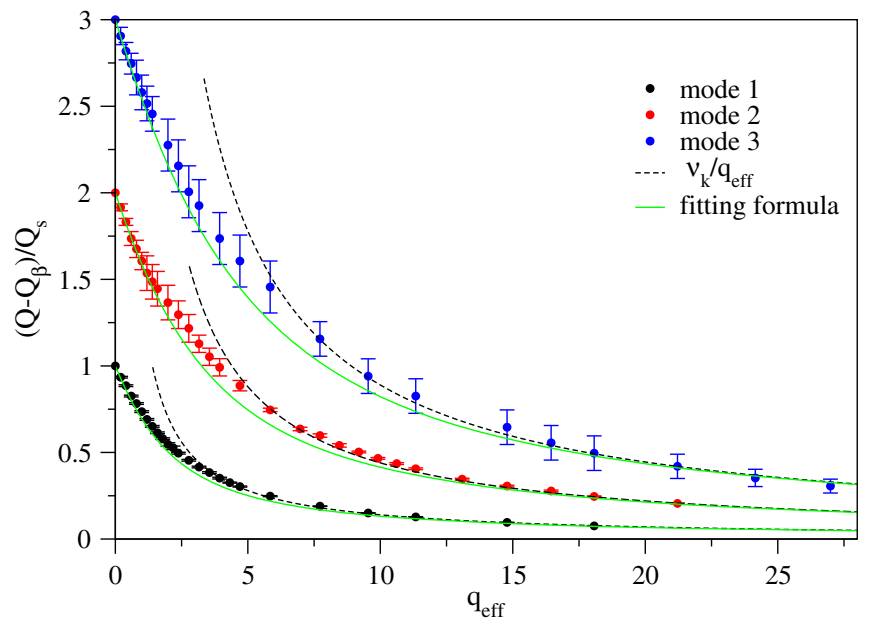

FIG. 7. 3D-G bunch. The relative tune shift, $\frac{Q-Q_{\beta}}{Q_{s}}$, for the modes 1,2 , and 3 versus the space charge parameter $q_{\text {eff }}$. In the strong space charge regime, $q>\approx 4 k, \frac{\Delta Q_{k}}{Q_{s}} \approx \frac{\nu_{k}}{q_{\text {eff }}}$, in good agreement with the theoretical prediction. $\nu_{1}=1.4, \nu_{2}=4.4$, and $\nu_{3}=8.9[6]$. The tune shift can be fitted reasonably well for the entire range of the space charge strength by employing Eq. (34) (green lines). 
tune shift equation derived by Blaskiewicz [7] for bunches with an airbag distribution in a square well potential. The equation for the airbag model in a square well potential is recovered by setting the coefficients $A_{k}=1$.

At small $q_{\text {eff }}$ the space charge harmonics are not a good approximation for the modes. Therefore the excitations with shapes proportional to the space charge harmonics excite multiple modes. For example, at $q_{\text {eff }}=0$, the first space charge harmonic predominantly excites modes with $m= \pm 1$. Other modes are excited too, but with a much smaller amplitude. The second space charge harmonic predominantly excites modes with $m= \pm 2$ and $m=0$. The third space charge harmonic predominantly excites modes with $m= \pm 3$ and modes with $m= \pm 1$ with various radial distribution. For purposes of illustration, in Fig. 8(a) we show the damping of the modes with large amplitude and the smallest damping rate excited with the first and the second space charge harmonics shapes. The corresponding tune shifts are shown in Fig. 8(b). The modes corresponding to $m=1$ and $m=2$ in the figure are the ones that transform into the space charge modes 1 and 2 with increasing $q_{\text {eff }}$. The other modes are more strongly damped.

Since at small $q_{\text {eff }}$ the radial modes are nearly degenerate, investigating the damping dependence as a function of the radial distribution and angular number $m$ is difficult there. The statistical noise can easily mix the modes. We believe the accuracy can be increased with higher statistics and by using excitation shapes proportional to modes at small $q_{\text {eff }}$. An exhaustive study of the modes in the small $q_{\text {eff }}$ regime requires further investigation.

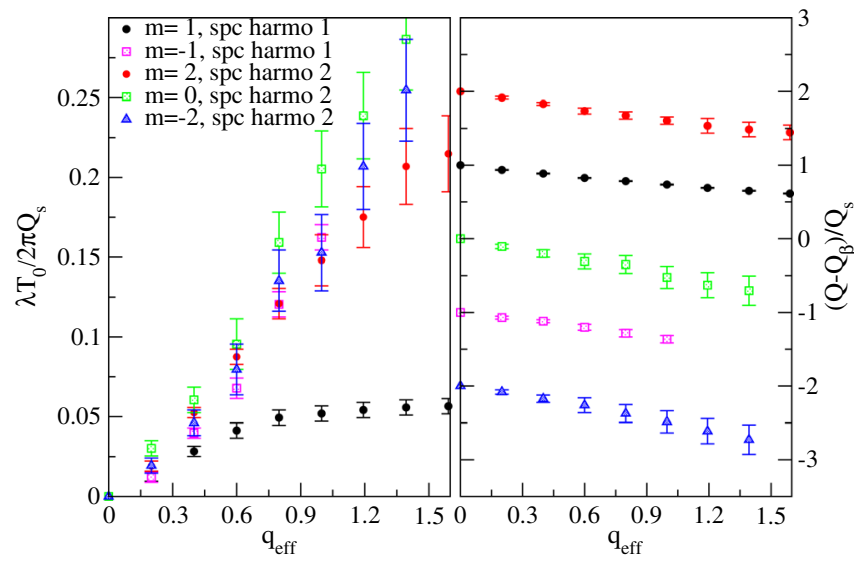

FIG. 8. 3D-G bunch. The Landau damping (a) and the relative tune shift (b) versus the space charge parameter $q_{\text {eff }}$ in the weak space charge regime for a few modes. The modes $m=-1,1$ in the figure are excited with a shape proportional to the first space charge harmonic while the modes $m=-2,0,2$ are excited with a shape proportional to the second space charge harmonic. $m$ corresponds to the angular number at $q_{\text {eff }}=0$. Only the modes which have a large overlap with the excitation shapes are shown. The modes which transform into the space charge modes with increasing $q_{\text {eff }}$ (black and red) are least damped.

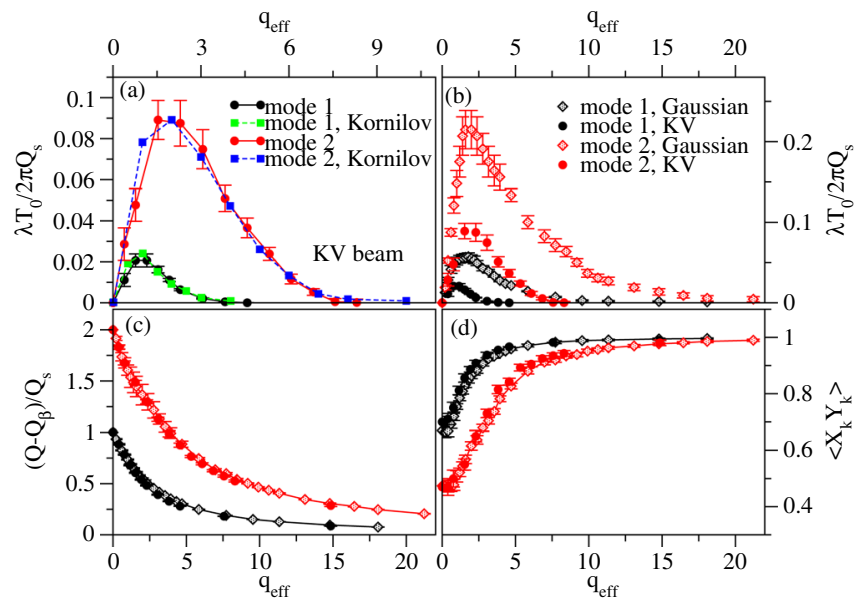

FIG. 9. (a) Landau damping for KV-G bunches. Comparison between our simulations and those of Kornilov and BoineFrankenheim [9]. The agreement is good. (b)-(d) Comparison between the first two modes of KV-G beams and 3D-G beams. (b) Landau damping. The damping of the 3D-G beams' modes is much larger. (c) Relative tune shift. (d) Spatial overlap of the mode shape with the space charge harmonic [see Eq. (32)]. Unlike the Landau damping the tune shift and the modes shapes depend very little on the transverse beam distribution, as expected.

\section{B. Transverse K-V distribution (KV-G)}

Employing particle tracking simulations, Kornilov and Boine-Frankenheim calculated the damping rates of longitudinally Gaussian beams with transverse KV distribution [9]. In Fig. 9(a) we compare the Landau damping of the first two modes obtained in our simulations for the KV-G beams (black and red circles) with the ones from Fig. 3 of Ref. [9] (green and blue squares). Despite the different approximations involved in the simulations, the agreement is good.

There is a significant difference between the damping rates of transverse Gaussian beams and transverse $\mathrm{KV}$ beams, as can be seen from Fig. 9(b). The difference is larger at large $q_{\text {eff }}$. In the strong space charge regime the damping for the $3 \mathrm{D}-\mathrm{G}$ beams is more than one order of magnitude larger than the damping for the KV-G beams.

Unlike the Landau damping, which is very sensitive to the transverse beam distribution, the tune shift and the modes spatial shapes show very small difference between the 3D-G and the KV-G distributions, in agreement with the theory [6]. That can be seen in Figs. 9(c) and 9(d) where the tune shift and the overlap with the space charge harmonics [see Eq. (32)] are shown for both beam distributions.

\section{DISCUSSION}

We calculated the momentum function defined as

$$
M_{k}(s)=\int X(z, u, s) Y_{k}(z) \rho(z) d z d u
$$


where $Y_{k}(z)$ is the space charge harmonic $k$, which is similar to the method employed in Ref. [9] for extracting the modes' properties. The damping values obtained from the analysis of the $M_{k}$ behavior are consistent with the ones obtained from the DMD analysis, although the results DMD are more accurate.

We want to point out several potential problems with the $M_{k}$ analysis. The analysis assumes that after the beam is excited with an approximate mode shape, only one mode remains after a sufficient time, all others being exponentially damped. This assumption is justifiable in the strong space charge regime where good approximations for the excitations are known from analytical calculations. However it is questionable in the intermediate region where the modes' shapes are unknown, especially for the higherorder modes. Moreover, when the Landau damping is not in the linear regime, the interpretation of data under this assumption might easily lead to wrong conclusions, as explained below.

It is essential to make sure the dynamics are in the linear damping regime. This can be seen in Fig. 10 where $M_{1}$ is shown for different values of the initial excitation amplitude at $q_{\text {eff }}=6$ for a 3D-G bunch. The linear regime requires an excitation amplitude smaller than $0.01 \sigma_{x}$. In the linear regime the $M_{1}$ envelope decays exponentially, with a damping rate equal to that provided by DMD. With larger initial excitations, the first 400 turns show similar decaying behavior. However, after longer times, the behavior is very different, with the envelope of $M_{1}$ exhibiting a slow beating, a behavior characteristic for nonlinear Landau damping [25]. For example, by analyzing the data with an amplitude excitation of $0.02 \sigma_{x}$ (red line), one can wrongly

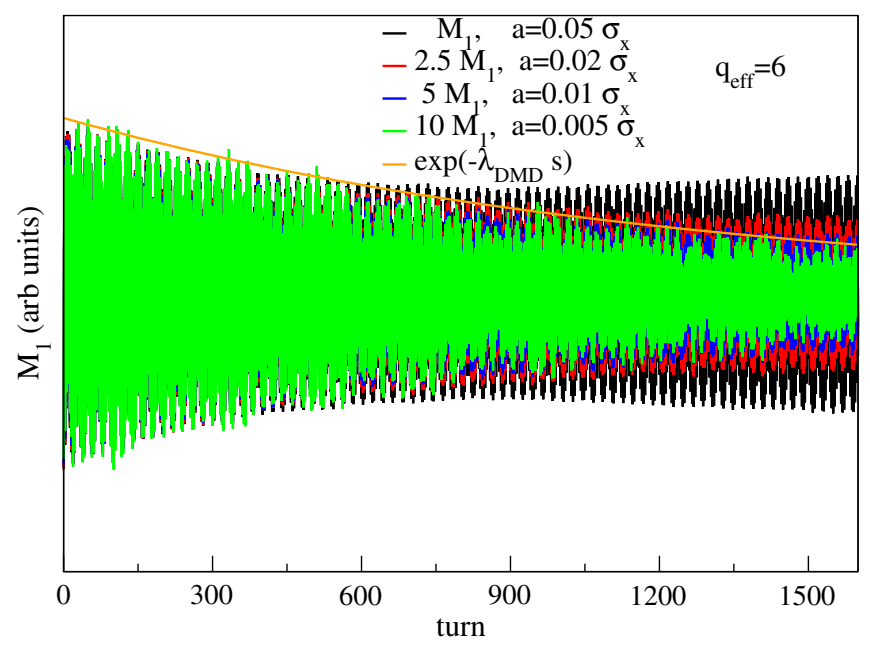

FIG. 10. 3D-G bunch. $M_{1}$, Eq. (36), versus turn number at $q_{\text {eff }}=6$ for different excitation amplitudes. $M_{1}$ is multiplied by a factor inverse proportional to the excitation amplitude. The linear regime requires an excitation amplitude smaller than $0.01 \sigma_{x}$. The exponential decay in the linear regime is consistent with the one provided by the DMD analysis. In the nonlinear regime the longtime behavior is very sensitive to the excitation amplitude. conclude that the behavior of the first 400 turns of $M_{1}$ shows the exponential decay of the high order modes while the long-time behavior yields zero Landau damping of the first mode. We find that at larger $q_{\text {eff }}$, the excitation amplitude must be reduced in order to satisfy the linear regime requirement.

Sufficient particle statistics is important for mode analysis. The simulation poses specific challenges over the entire interaction range. At small $q_{\text {eff }}$ there is a strong degeneracy of modes that makes the mode mixing susceptible to statistical noise. The intermediate $q_{\text {eff }}$ region is subject to strong damping, which causes the damped signal to approach the noise level quite rapidly. At large $q_{\text {eff }}$ the space charge nonlinear effects are strong, requiring a small excitation amplitude for the linear Landau damping regime. But a small excitation amplitude implies a small signal-tonoise ratio. Our simulations used $10^{7}$ macroparticles.

The results shown here are obtained for the synchrotron tune $Q_{s}=0.01$. The simulations with $Q_{s}=0.005$ produce consistent results.

\section{SUMMARY AND CONCLUSIONS}

A numerical investigation of the transverse modes and their intrinsic Landau damping in Gaussian bunched beams with space charge was presented. The beam dynamics is simulated with the SYNERGIA accelerator simulation package. The dynamic mode decomposition technique is used to extract the properties of the modes from the simulated data.

As the strength of the space charge is increased, the modes change gradually from the radially degenerate phase space harmonics to the momentum-independent space charge harmonics of Refs. [6,8]. Based on the evolution of the mode shape, damping and tune shift with increasing $q_{\text {eff }}$, we estimate $q_{\text {eff }} \approx 4 k$ to be the threshold value which defines the strong space charge regime. This is in agreement with the theoretical requirement $q_{\text {eff }} \gg 2 k$, where $k$ is the mode number.

Comparison between the mode properties of bunches with Gaussian transverse distributions and of those with $\mathrm{KV}$ transverse distributions shows that the damping is very sensitive to the bunch transverse distribution shape, even though the tune shifts and mode shapes themselves depend very little on the transverse shape, which also agrees with the theoretical predictions.

In the strong space charge regime the damping for the $3 \mathrm{D}-\mathrm{G}$ beams is $\lambda_{k} T_{0} \approx 2.4 \frac{k^{4} 2 \pi Q_{s}}{q_{\text {eff }}^{\text {sf }}}$, while for KV-G beams it is much smaller. The tune shift of the modes is in agreement with the theoretical predictions and equal to $\frac{\nu_{k} Q_{s}}{q_{\text {eff }}}$, where $\nu_{k}$ constants are tabulated in Ref. [6]. The damping reaches a maximum in the intermediate region for $q_{\text {eff }} \approx 2 k$. The maximum damping increases with the mode number, as expected. Formulas for the damping rates and the tune shifts that fit the numerical results reasonably well for the entire range of the space charge strengths were proposed. 
At $q_{\text {eff }}=0$ the modes are radially degenerate, being characterized by the azimuthal harmonic number $m$ and tune shift $m Q_{s}$. The space charge mode $k$ transforms into a mode with angular number $m=k$ upon decreasing $q_{\text {eff }}$. The space charge interaction lifts the degeneracy of the noninteracting regime. However, in the weakly interacting regime there are a large number of modes with closely spaced frequencies, making the numerical investigation of this regime difficult. An exhaustive study of the modes in the weakly interacting regime requires further investigation. Here we only considered the modes which are significantly excited by the space charge harmonics. Among these modes, those which evolve into the space charge modes upon increasing $q_{\text {eff }}$, i.e., the ones with the shape closest to the space charge harmonic, have the smallest damping rates.

\section{ACKNOWLEDGMENTS}

This work was performed at Fermilab, operated by Fermi Research Alliance, LLC under Contract No. DE-AC0207CH11359 with the U.S. Department of Energy. SYNERGIA development is partially supported through the ComPASS project, funded through the Scientic Discovery through Advanced Computing program in the DOE Office of High Energy Physics. An award of computer time was provided by the Innovative and Novel Computational Impact on Theory and Experiment (INCITE) program. This research used resources of the Argonne Leadership Computing Facility, which is a DOE Office of Science User Facility supported under Contract No. DE-AC02$06 \mathrm{CH} 11357$.

[1] D. Mohl and H. Schonauer, Proceedings of the IX International Conference on High Energy Accelerators, Stanford (AEC, Washington, D.C., 1974), p. 380.

[2] G. Merle and D. Mohl, Report No. CERN/ISR/300/GS/6966, 1969.

[3] V. I. Balbekov, Sov. Phys. Tech. Phys. 21, 837 (1976).

[4] L. Landau, Oscillations of electron plasma, J. Phys. USSR 10, 25 (1946).

[5] A. Burov and V. Lebedev, Transverse instabilities of coasting beams with space charge, Phys. Rev. ST Accel. Beams 12, 034201 (2009).

[6] A. Burov, Head-tail modes for strong space charge, Phys. Rev. ST Accel. Beams 12, 044202 (2009); 12, 109901(E) (2009).
[7] M. Blaskiewicz, Fast head-tail instability with space charge, Phys. Rev. ST Accel. Beams 1, 044201 (1998).

[8] V. Balbekov, Transverse modes of a bunched beam with space charge dominated impedance, Phys. Rev. ST Accel. Beams 12, 124402 (2009).

[9] V. Kornilov and O. Boine Frankenheim, Head-tail instability and Landau damping in bunches with space charge, Phys. Rev. ST Accel. Beams 13, 114201 (2010).

[10] V. Kornilov et al., Proceedings of HB2014 ICFA Workshop, East-Lansing, MI, USA (2014), WEO1LR02, http:// epaper.kek.jp/HB2014.

[11] V. Kornilov (private communication).

[12] Synergia2, Accelerator Modeling, https://cdcvs.fnal.gov/ redmine/projects/synergia2/wiki.

[13] J. Amundson, P. Spentzouris, J. Qiang, and R. Ryne, Synergia: An accelerator modeling tool with 3-D space charge, J. Comp. Physiol. 211, 229 (2006).

[14] P. J. Schmid, Dynamic mode decomposition of numerical and experimental data, J. Fluid Mech. 656, 5 (2010).

[15] C. W. Rowley, I. Mezić, S. Bagheri, P. Schlatter, and D. S. Henningson, Spectral analysis of nonlinear flows, J. Fluid Mech. 641, 115 (2009).

[16] J.H. Tu et al., On dynamic mode decomposition: Theory and applications, J. Comput. Dynam. 1, 391 (2014).

[17] K. K. Chen, J.H. Tu, and C. W. Rowley, Variants of dynamic mode decomposition: Boundary condition, Koopman, and Fourier analyses, J. Nonlinear Sci. 22, 887 (2012).

[18] See Ref. [16] and references therein.

[19] B. W. Brunton et al., Extracting spatial-temporal coherent patterns in large-scale neural recordings using dynamic mode decomposition, arXiv:1409.5496.

[20] J. Grosek et al., Dynamic mode decomposition for real-time background/foreground separation in video, arXiv:1404.7592.

[21] A. Chao, Physics of Collective Beam Instabilities in High Energy Accelerators (John Wiley \& Sons, Inc., New York, 1993), Eq. (6.170) with $\xi=0$ and $F_{y}=0$.

[22] Note the $2 \pi$ factor difference between our definition of $\lambda T_{0}$ and the definition of $\lambda$ in Ref. [6]. While we consider the damping per turn, in Ref. [6] the damping is expressed in $\omega=\frac{2 \pi}{T_{0}}$ units.

[23] H. Yoshida, Construction of higher order symplectic integrators, Phys. Lett. A 150, 262 (1990).

[24] R. W. Hockney and J. W. Eastwood, Computer Simulation Using Particles (McGraw-Hill, New York, 1981).

[25] T. Zhou, Y. Guo, and C.-W. Shu, Numerical study on Landau damping, Physica D (Amsterdam) 157, 322 (2001). 